\title{
HPV vaccination among females with mental and physical limitation
}

Melissa Rowe, MD ${ }^{1}$, Audra L Pritt, MD1, Amanda J. Stratton, MSIV ${ }^{1}$, Jennie L. Yoost, MD, $\mathrm{MSc}^{1}$

\section{Author Affiliations:}

1. Joan C. Edwards School of Medicine, Marshall University, Huntington, West Virginia

The authors have no conflicts of interest to disclose.

\section{Corresponding Author:}

Jennie L. Yoost, MD, MSc

Department of Obstetrics \& Gynecology

Marshall University

Joan C. Edwards School of Medicine

1600 Medical Center Drive

Huntington, West Virginia 25701

Email: yoost@marshall.edu 


\section{Abstract}

Introduction

Adolescent HPV vaccination rates lag behind other vaccinations, partly due to missed opportunities for vaccination and lack of provider recommendation. This study evaluated the rates of HPV vaccination among adolescents with physical and mental limitation and controls, and evaluated differences in parental knowledge and reasons for nonvaccination between groups.

\section{Materials and Methods}

A retrospective chart review was performed on female patients age 12-18 presenting to pediatric or gynecology clinics in 2012. Those subjects with a diagnosis of mental or physical limitation were classified as "affected". HPV vaccination rates were compared between affected and control groups. Parents of affected subjects were contacted by phone and surveyed regarding HPV knowledge and reasons for nonvaccination. Parents of controls were contacted at a 2:1 ratio and were administered the same survey.

Results

1673 females were included in the study; 72 (4.3\%) had mental/physical impairment (affected). Overall, 944 (56.4\%) subjects had initiated HPV vaccination and 653 (39\%) had completed the three dose series. When comparing affected subjects to controls, the control group was more likely to have received any HPV vaccination (RR 1.32, 95\% CI 1.013, 1.73). Parents of 21 affected subjects and 44 control subjects were contacted by phone. No differences were found in parent knowledge of HPV or importance of vaccination to a child's health. There were no differences in reasons for nonvaccination between groups, but for both groups "lack of provider recommendation" was in the top two choices. That the vaccine was "not needed" was the other main reason given for nonvaccination in the affected group, while the control group main reason for nonvaccination was "concerns for safety".

\section{Discussion}

Adolescents with mental or physical limitation may be less likely to receive HPV vaccination. Lack of provider recommendation remains one of the primary reasons those in this population do not receive HPV vaccination.

\section{Keywords}

Human papillomavirus, vaccination, mental limitation, physical limitation

\section{Introduction}

Human papillomavirus (HPV) is associated with the development of anogenital cancers such as cervical, vaginal, vulvar, penile and anal. HPV also causes genital warts and certain types of oropharyngeal cancer. ${ }^{1}$ The Advisory Committee on Immunization Practices recommends HPV vaccination for girls and boys at the target age of 11-12, with catch up vaccination up to age 26 . The vaccine was initially recommended for all ages as a series of three injections over a six month period of time. ${ }^{2}$ In 2016, these recommendations changed to a two dose series for those initiating vaccination under age $15 .^{3}$ HPV vaccination has been demonstrated to be safe and effective at reducing the risk of genital warts and HPV associated cancers. ${ }^{4,5}$ Despite this demonstrated safety and efficacy, in $2015,62.8 \%$ of females in a nationwide sample received at 
least one HPV vaccination, with only $41.9 \%$ receiving all three injections. ${ }^{6}$. While these numbers have increased from recent years, HPV vaccination still lags behind other vaccine uptake and the Healthy People target of $80 \% .^{6}$ In one national survey, the most common reasons for parents choosing not to vaccinate against HPV were 1) they felt the vaccine is not needed, 2) their child is not sexually active, 3) parental concerns about safety or side effects, 4) lack of knowledge about the vaccine, and 5) the vaccine was not recommended by their provider. ${ }^{7}$

HPV vaccination recommendations include those children and adolescents with special health care needs. It was our hypothesis that among children with physical and mental limitation, HPV vaccination would be lower than among children without these limitations. One small study demonstrated that parents of children with special health care needs have difficulty accepting the HPV vaccine primarily due to the perception that their child was unlikely to be sexually active. ${ }^{8}$ However, another study including a sample of $>20,0007-12^{\text {th }}$ grade females concluded that those with mobility impairments and those with learning disabilities are just as likely as their peers to be sexually active. ${ }^{9}$ It is also known that adolescents with disabilities are at increased risk of being sexually abused compared to the general population. In one study, $16.6 \%$ of children with autism had been sexually abused per report from caregivers. ${ }^{10}$

This study evaluated the rates of HPV vaccination among adolescents with physical and mental limitation compared to adolescents without these limitations. The aim was to evaluate if vaccination rates were different, and if reasons for nonvaccination were different between the two groups.

\section{Materials and Methods}

This study is a retrospective chart review and cross sectional survey that assessed the vaccine rate among female patients with mental and physical limitation compared to those without these limitations. Charts of female patients ages 12-18 years presenting for well child visits or routine health maintenance visits from January 1, 2012 through December 31, 2012 were reviewed. This review included female patients from the pediatric and gynecology clinics at an academic institution. The chart review and survey were conducted in 2015. The study year of 2012 was chosen in order to give those patients presenting at the end of the year adequate time for vaccine series completion. Only females were included in this study, as the HPV vaccine recommendation for males was not made routine until 2011. Patients reviewed from the gynecology clinics were nonpregnant adolescents within the specified age group.

Patients were identified by using CPT codes for well child visits. Data was collected on the patients' age, diagnoses, number of vaccinations in the series, and dates of vaccinations. Mental and physical delays were determined by a chart review using the patients past medical history and diagnosis codes. Those subjects with either a diagnosis of mental or physical limitation are referred to as the "affected" group. These patient diagnoses were recorded and discussed among the research team. Those patients agreed as having a diagnosis consistent with mental or physical limitation were placed in the affected group. Patients without diagnoses of mental of physical limitation were placed in the control group. Rates of HPV vaccination among the affected group and control group were compared.

Parents or guardians of those subjects in the affected group not receiving HPV vaccination were then contacted by phone and a short survey was conducted. Up to three attempts were made to contact each of the unvaccinated subjects' parent in the affected group. The phone survey lasted 
five minutes and included single answer questions such as true/false questions regarding knowledge of HPV transmission and vaccination. The phone survey also included parental perception of vaccine safety and importance measured by Likert scale. Reasons for nonvaccination against HPV were also assessed by multiple choice based on the most cited reasons of 1) not needed or necessary, 2) child not sexually active, 3) concerns for safety or side effects, 4) lack of knowledge about the vaccine, and 5) not recommended by medical provider. For this question, they were asked to cite the single main reason why their child did not receive the HPV vaccine.

Parents of subjects in the unvaccinated control group were also contacted by phone for the same survey assessment. As it was not feasible to contact the entire control population, control subjects were age matched in a 2:1 ratio to the affected group for comparison. Parents or guardians of this control group were contacted using the same survey. Vaccine knowledge, reasons for nonvaccination, and perception of vaccine safety and importance were compared between groups. Between group data was analyzed comparing proportions using Pearson Chi Square or Fishers exact test when appropriate. The T-test was used for continuous variables. SPSS version 22 was used for all statistical analyses. Institutional Review Board approval was obtained prior to the study.

\section{Results}

The chart review yielded 1673 females that met criteria for well child visits presenting to either the pediatric or gynecology clinics during the designated study period. Of these females, 72 $(4.3 \%)$ were found to have mental/physical impairment. These subjects included diagnoses such as cerebral palsy, Rett syndrome, Noonan syndrome, autism, Down Syndrome, Asperger syndrome, arthrogryposis, muscular dystrophy, and mental retardation. Overall, 944 (56.4\%) subjects had initiated HPV vaccination and $653(39 \%)$ had completed the three dose series. The control group age was slightly older than the affected group age during the study period (15.9 (SD 2.7) vs 14.8 (SD 2.4); $\mathrm{p}=0.058$ ). However, the mean age at first HPV injection did not differ between control and affected group (12.2 (SD 4.0) vs 12.2 (SD 1.8); $\mathrm{p}=0.79$ ).

In the control group, 913 subjects out of 1601 (57\%) had at least one HPV vaccination documented. In the affected group, 31 subjects out of $72(43 \%)$ had at least one HPV vaccination documented. When comparing affected subjects to controls, the control group was more likely to have received any HPV vaccination (RR $1.3295 \%$ CI 1.013, 1.73, Table 1). Among those that had initiated vaccination, similar percentages were seen between groups of those completing all three doses $(22$ (79.9\%) affected vs $631(69 \%)$ controls; $p=0.83)$.

Table 1: Vaccination rates between affected and control groups

\begin{tabular}{|c|c|c|c|}
\hline & Vaccinated & Not Vaccinated & \\
\hline Affected Group (n=72) & $31(43 \%)$ & $41(57 \%)$ & \\
\hline Control Group $(n=1601)$ & $913(57 \%)$ & $688(43 \%)$ & RR $1.32(1.013,1.73)$ \\
\hline
\end{tabular}

There were 41 out of 72 affected subjects that did not receive any vaccine. Of these subjects only 21 parents $(51.2 \%)$ were able to be contacted by phone survey. Out of the 688 controls not 
vaccinated, subjects were age matched to the affected group in a 2:1 ratio. Survey data was collected from a resultant 44 unvaccinated control parents. HPV knowledge was similar among parents of the affected group and controls, except for the True/False question "HPV causes cough", which was answered incorrectly by more parents in the control group (Table 2). Only $33.3 \%$ of parents in the affected group and $45.5 \%$ of parents in the control group answered correctly that "HPV causes genital warts". Awareness increased that "HPV causes cervical cancer" with $81 \%$ of affected and $72.7 \%$ of control group answering correctly. However, awareness of oropharyngeal cancers linked to HPV was low in both groups $(28.6 \%$ of affected and $20.5 \%$ of controls). The majority in both groups were aware that HPV was transmitted by sexual activity.

Table 2: Comparison of HPV knowledge between affected and control groups. Responses listed

\begin{tabular}{|c|c|c|c|c|c|}
\hline \multirow[t]{2}{*}{ True/False Question } & \multicolumn{2}{|c|}{ Affected Group $(n=21)$} & \multicolumn{2}{|c|}{ Controls $(n=44)$} & \multirow{2}{*}{$\begin{array}{l}\mathbf{p} \\
\text { value }\end{array}$} \\
\hline & $\%$ Yes & $\%$ No & $\%$ Yes & $\%$ No & \\
\hline $\begin{array}{l}\text { HPV causes cough } \\
\text { (False) }\end{array}$ & $0(0)$ & $100(21)$ & $31.8(14)$ & $68.2(30)$ & 0.003 \\
\hline $\begin{array}{l}\text { HPV causes genital warts } \\
\text { (True) }\end{array}$ & $33.3(7)$ & $66.7(14)$ & $45.5(20)$ & $54.5(24)$ & 0.354 \\
\hline $\begin{array}{l}\text { HPV causes cervical cancer } \\
\text { (True) }\end{array}$ & $81(14)$ & $19(4)$ & $72.7(32)$ & $27.3(12)$ & 0.472 \\
\hline $\begin{array}{l}\text { HPV causes heart attacks } \\
\text { (False) }\end{array}$ & $9.5(2)$ & $90.5(19)$ & $22.7(10)$ & $77.3(34)$ & 0.309 \\
\hline $\begin{array}{l}\text { HPV causes throat cancer } \\
\text { (True) }\end{array}$ & $28.6(6)$ & $71.4(15)$ & $20.5(9)$ & $79.5(35)$ & 0.535 \\
\hline $\begin{array}{l}\text { People can have HPV and } \\
\text { have no symptoms } \\
\text { (True) }\end{array}$ & $61.9(13)$ & $38.1(8)$ & 43.2 (19) & $56.8(25)$ & 0.158 \\
\hline $\begin{array}{l}\text { People can get HPV from } \\
\text { sexual activity } \\
\text { (True) }\end{array}$ & $100(21)$ & $0(0)$ & $84.1(37)$ & $15.9(7)$ & 0.086 \\
\hline $\begin{array}{l}\text { People can get HPV from } \\
\text { someone coughing or } \\
\text { sneezing on them } \\
\text { (False) }\end{array}$ & $4.8(1)$ & $95.2(20)$ & $15.9(7)$ & $84.1(37)$ & 0.259 \\
\hline $\begin{array}{l}\text { People can get HPV from } \\
\text { undercooked meat } \\
\text { (False) }\end{array}$ & $4.8(1)$ & $95.2(20)$ & $18.2(8)$ & $81.8(36)$ & 0.251 \\
\hline
\end{tabular}

There were no differences in chosen reasons not to vaccinate their child between groups (Table 3). Among parents in the affected group, the two most commonly cited reasons were that the vaccine was not needed $(n=7)$ and that the vaccine was not recommended by their medical provider $(n=7)$. In the control group, the two most cited reasons were safety concerns $(n=15)$ and lack of recommendation from their provider $(n=10)$. 
Table 3: Reasons chosen for HPV nonvaccination compared between groups.

\begin{tabular}{llll}
\hline Reason & Affected $(\mathbf{n}=\mathbf{2 1})$ & Control $(\mathbf{n}=\mathbf{4 4})$ & p value \\
\hline Not needed & $33.3 \%(7)$ & $15.9 \%(7)$ & 0.195 \\
Child not sexually active & $9.5 \%(2)$ & $15.9 \%(7)$ & 0.706 \\
Concern for safety or side effects & $19 \%(4)$ & $34.1 \%(15)$ & 0.256 \\
Lack of knowledge about the vaccine & $4.8 \%(1)$ & $11.4 \%(5)$ & 0.655 \\
Not recommended by medical provider & $33.3 \%(7)$ & $22.7 \%(10)$ & 0.381 \\
\hline
\end{tabular}

Thirty four (77.3\%) of the control group and 17 (81\%) of affected group felt that vaccines were very important to their child's health $(\mathrm{p}=0.736)$. Those that felt vaccines were "very safe" and "somewhat safe" were also comparable between control and affected groups: (19 (43.2\%) vs 11 $(52.4 \%) ; \mathrm{p}=0.487$ and $22(50 \%)$ vs $9(42.9 \%) ; \mathrm{p}=0.59$, respectively, Table 4$)$.

Table 4: Comparison of parent responses to Likert scale questions of "How important are vaccines to your child's health?", and "In general, how safe do you think vaccines are?".

\begin{tabular}{llll}
\hline Response & Affected $(\mathbf{n}=\mathbf{2 1})$ & Control $(\mathbf{n}=\mathbf{4 4})$ & p value \\
\hline Very important & $81 \%(17)$ & $77.3 \%(34)$ & 0.736 \\
\hline Very Safe & $52.4 \%(11)$ & $43.2 \%(19)$ & 0.487 \\
\hline Somewhat safe & $42.9 \%(9)$ & $50 \%(22)$ & 0.59 \\
\hline
\end{tabular}

\section{Discussion}

In this study we found that females with mental or physical limitation are less likely to receive HPV vaccination compared to those without these limitations. Our study population had overall comparable rates of HPV vaccine initiation (56.4\%) and completion (39\%) compared to national reported rates. Among those initiating vaccination, we also found similar completion rates between the two groups. While no significant differences between groups were found with parental knowledge and reasons for nonvaccination, this affected population may have other barriers not elucidated by this study design.

This study is limited by the overall number of subjects included. Our affected subjects comprised $4.3 \%$ of the total study population, which is similar to percentages in other studies looking at adolescents within a population with physical or mental limitation. ${ }^{9}$ Due to the small number of surveys able to be completed on the affected population, we may not have had enough power to detect any differences from the control group. A 2:1 matching approach was done on the control group to collect enough surveys for comparison. This was done as collecting phone surveys on all 688 unvaccinated controls was not feasible.

The retrospective chart design also adds limitation to study. While each chart was reviewed and the research team agreed whether or not to classify a patient as "affected" or not, this approach is somewhat subjective. Other factors such as insurance status, parental age, and subject sexual activity were not collected, but all could affect vaccine adherence or relevance. Parental knowledge was assessed on HPV vaccination, but education level and knowledge of other vaccinations was not obtained. 
Despite these limitations, there is paucity of data regarding HPV vaccination among adolescents with special needs. The only contraindication to HPV vaccination is allergic reaction to any component of the vaccine. ${ }^{2}$ Furthermore, it is important to recognize that HPV transmission can occur in a variety of ways including hand transmission ${ }^{11}$, and HPV can be found in children prior to any sexual activity. ${ }^{12}$ For these reasons, all adolescents should receive HPV vaccination at the recommended age regardless of past medical history. In females with special needs, cervical cancer screening may also prove more difficult, making HPV vaccination a priority.

In both control and affected populations, parents placed high importance on vaccines in regards to their child's health, and felt that vaccines were overall safe. However, this contradicts reasons that they gave for nonvaccinaction such as "concern for safety" or "not needed". It is possible that this is because parents may view HPV vaccination differently from other scheduled vaccines. In both populations, lack of a recommendation from a medical provider was one of the primary reasons for not getting their child vaccinated. Provider attitudes were not assessed in our study; however, a prior study found that providers caring for females with special needs placed a high priority on recommended vaccinations. ${ }^{8}$ It has been well documented that a provider recommendation is the most influential factor in a patient's decision to receive an immunization, and that more parents of vaccinated teens reported receiving a recommendation compared with parents of unvaccinated teens. ${ }^{13}$

It is important to recognize populations at risk for missed vaccination opportunities. HPV vaccination should be recommended to all adolescents at the target age, including those with physical and mental limitation. Future directions would include study on improving provider recommendations, and provider and parent awareness of HPV vaccination in underserved populations such as those with mental and physical limitation. 


\section{References}

1. Markowitz LE, Dunne EF, Saraiya M, Chesson HW, Curtis CR, Gee J, et al. Human papillomavirus vaccination: recommendations of the Advisory Committee on Immunization Practices (ACIP). MMWR Recommendations and reports : Morbidity and mortality weekly report Recommendations and reports / Centers for Disease Control. 2014 Aug 29;63(RR-05):1-30. PubMed PMID: 25167164.

2. Robinson CL, Advisory Committee on Immunization Practices ACAIWG. Advisory Committee on Immunization Practices Recommended Immunization Schedules for Persons Aged 0 Through 18 Years-United States, 2016. MMWR Morbidity and mortality weekly report. 2016;65(4):86-7. PubMed PMID: 26845283.

3. Meites E, Kempe A, Markowitz LE. Use of a 2-Dose Schedule for Human Papillomavirus Vaccination Updated Recommendations of the Advisory Committee on Immunization Practices. MMWR Morbidity and mortality weekly report. 2016 Dec 16;65(49):1405-8. PubMed PMID: 27977643.

4. Munoz N, Kjaer SK, Sigurdsson K, Iversen OE, Hernandez-Avila M, Wheeler CM, et al. Impact of human papillomavirus (HPV)-6/11/16/18 vaccine on all HPV-associated genital diseases in young women. Journal of the National Cancer Institute. 2010 Mar 3;102(5):325-39. PubMed PMID: 20139221.

5. Gee J, Naleway A, Shui I, Baggs J, Yin R, Li R, et al. Monitoring the safety of quadrivalent human papillomavirus vaccine: findings from the Vaccine Safety Datalink. Vaccine. 2011 Oct 26;29(46):8279-84. PubMed PMID: 21907257.

6. Reagan-Steiner S, Yankey D, Jeyarajah J, Elam-Evans LD, Curtis CR, MacNeil J, et al. National, Regional, State, and Selected Local Area Vaccination Coverage Among Adolescents Aged 13-17 Years - United States, 2015. MMWR Morbidity and mortality weekly report. 2016;65(33):850-8. PubMed PMID: 27561081.

7. Dorell C, Yankey D, Kennedy A, Stokley S. Factors that influence parental vaccination decisions for adolescents, 13 to 17 years old: National Immunization Survey-Teen, 2010. Clinical pediatrics. 2013 Feb;52(2):162-70. PubMed PMID: 23221308.

8. Cody PJ, Lerand SJ. HPV vaccination in female children with special health care needs. Journal of pediatric and adolescent gynecology. 2013 Aug;26(4):219-23. PubMed PMID: 23726139.

9. Blum RW, Kelly A, Ireland M. Health-risk behaviors and protective factors among adolescents with mobility impairments and learning and emotional disabilities. The Journal of adolescent health : official publication of the Society for Adolescent Medicine. 2001 Jun;28(6):481-90. PubMed PMID: 11377992.

10. Mandell DS, Walrath CM, Manteuffel B, Sgro G, Pinto-Martin JA. The prevalence and correlates of abuse among children with autism served in comprehensive community-based mental health settings. Child abuse \& neglect. 2005 Dec;29(12):1359-72. PubMed PMID: 16293306.

11. Sonnex C, Strauss S, Gray JJ. Detection of human papillomavirus DNA on the fingers of patients with genital warts. Sexually transmitted infections. 1999 Oct;75(5):317-9. PubMed PMID: 10616355. Pubmed Central PMCID: 1758241.

12. Bacopoulou F, Karakitsos P, Kottaridi C, Stefanaki C, Deligeoroglou E, Theodoridou K, et al. Genital HPV in Children and Adolescents: Does Sexual Activity Make a Difference? Journal of pediatric and adolescent gynecology. 2016 Jun;29(3):228-33. PubMed PMID: 26342734.

13. Stokley S, Jeyarajah J, Yankey D, Cano M, Gee J, Roark J, et al. Human papillomavirus vaccination coverage among adolescents, 2007-2013, and postlicensure vaccine safety monitoring, 2006-2014--United States. MMWR Morbidity and mortality weekly report. 2014 Jul 25;63(29):620-4. PubMed PMID: 25055185 . 